\title{
Monopoles in an Antiferromagnetic Bose-Einstein Condensate
}

\author{
H. T. C. Stoof, E. Vliegen, and U. Al Khawaja \\ Institute for Theoretical Physics, University of Utrecht, Princetonplein 5, 3584 CC Utrecht, The Netherlands
}

(Received 8 March 2001; published 3 September 2001)

\begin{abstract}
We show that even in three dimensions an antiferromagnetic spin-1 Bose-Einstein condensate, which can, for instance, be created with ${ }^{23} \mathrm{Na}$ atoms in an optical trap, has not only singular linelike vortex excitations, but also allows for singular pointlike topological excitations, i.e., monopoles similar to the 't Hooft-Polyakov monopoles. We discuss the static and dynamic properties of these monopoles.
\end{abstract}

DOI: $10.1103 /$ PhysRevLett.87.120407

Introduction.-Quantum magnetism plays an important role in such diverse areas of physics as high-temperature superconductivity, quantum phase transitions, and the quantum Hall effect. Moreover, it now appears that magnetic properties are also very important in another area, namely Bose-Einstein condensation in trapped atomic gases. This is due to two independent experimental developments. The first development is the realization of an optical trap for ${ }^{23} \mathrm{Na}$ atoms [1], whose operation no longer requires the gas to be doubly spin polarized and has given rise to the creation of a spin-1 Bose-Einstein antiferromagnet [2]. The second development is the creation of a two-component condensate of ${ }^{87} \mathrm{Rb}$ atoms [3], which by means of rf fields can be manipulated so as to make the two components essentially equivalent [4]. As a result also a spin-1/2 Bose-Einstein ferromagnet can now be studied in detail experimentally.

The spin structure of these condensates has recently been worked out by a number of authors [5-8] and also the first studies of the linelike vortex excitations have appeared $[5,9,10]$. An immediate question that comes to mind, however, is whether the spin degrees of freedom lead also to other topological excitations that do not have an analogy in the case of a single component Bose-Einstein condensate. The answer to this question is in general affirmative. Indeed, we have recently shown that ferromagnetic Bose-Einstein condensates have long-lived Skyrmion excitations, which are nonsingular but topologically nontrivial pointlike spin textures [11]. Moreover, we show here that also spin-1 Bose-Einstein antiferromagnets have pointlike topological excitations. In particular, there exist singular pointlike spin textures, which are analogous to the magnetic monopoles in particle physics discovered by 't Hooft and Polyakov [12]. Having done so, we then turn to the investigation of the precise texture and the dynamics of these monopoles.

As indicated above, Skyrmion and monopole excitations have already been studied in the context of nuclear and high-energy physics, respectively. However, in these areas of physics there does not exist a satisfactory microscopic theory for these topological excitations. For example, the Skyrme model gives only a rather rough description of a nucleon. Moreover, magnetic monopoles
PACS numbers: 03.75.Fi, 14.80.Hv, 32.80.Pj, 67.40.-w

have never been observed experimentally. Creating such excitations in Bose-Einstein condensed gases offers therefore the exciting opportunity to study, to the best of our knowledge for the first time in a quantum fluid, the properties of Skyrmions and monopoles in exquisite detail, both theoretically and experimentally. Undoubtedly this will lead to important new insights into the general topic of topological excitations in a quantum field theory.

Topological considerations. - To find the topological excitations of a spin-1 Bose-Einstein condensate, we need to know the full symmetry of the macroscopic wave function $\Psi(\mathbf{r}) \equiv \sqrt{n(\mathbf{r})} \zeta(\mathbf{r})$, where $n(\mathbf{r})$ is the total density of the gas, $\zeta(\mathbf{r})$ is a normalized spinor that determines the average local spin by means of $\langle\mathbf{F}\rangle(\mathbf{r})=\zeta^{\dagger}(\mathbf{r}) \mathbf{F} \zeta(\mathbf{r})$, and $\mathbf{F}$ are the usual spin matrices obeying the commutation relations $\left[F_{\alpha}, F_{\beta}\right]=i \epsilon_{\alpha \beta \gamma} F_{\gamma}$. Note that here, and in the following, summation over repeated indices is always implied. From the work of Ho [5] we know that in the antiferromagnetic case the mean-field interaction energy is minimized for $\langle\mathbf{F}\rangle(\mathbf{r})=\mathbf{0}$, which implies that the parameter space for the spinor $\zeta(\mathbf{r})$ is only $S^{1} \times S^{2}$ because we are free to choose both its overall phase and the orientation of the spin quantization axis. Introducing the superfluid phase $\vartheta(\mathbf{r})$ and the unit vector field $\mathbf{m}(\mathbf{r})$, this topology can also be understood explicitly from the fact that all the spinors

$$
\zeta(\mathbf{r})=\frac{e^{i \vartheta(\mathbf{r})}}{\sqrt{2}}\left(\begin{array}{c}
-m_{x}(\mathbf{r})+i m_{y}(\mathbf{r}) \\
\sqrt{2} m_{z}(\mathbf{r}) \\
m_{x}(\mathbf{r})+i m_{y}(\mathbf{r})
\end{array}\right) \equiv e^{i \vartheta(\mathbf{r})} \zeta_{\mathrm{AF}}(\mathbf{r})
$$

have a vanishing average spin and hence are locally degenerate. In quantum magnetism the vector $\mathbf{m}$ is known as the Néel vector and roughly speaking corresponds to the staggered magnetization of our itinerant antiferromagnet.

What does this tell us about the possible topological excitations? For linelike defects or vortices, we can assume $\zeta(\mathbf{r})$ to be independent of one direction and the spinor represents a mapping from a two-dimensional plane into the order parameter space. If the vortex is singular, this is visible on the boundary of the two-dimensional plane and we need to investigate the properties of a continuous mapping from a circle $S^{1}$ into the order parameter space $G$, i.e., of the first homotopy group $\pi_{1}(G)$ [13]. Since 
$\pi_{1}\left(S^{1} \times S^{2}\right)=Z$, we conclude that an antiferromagnetic spin-1 condensate can have vortices with winding numbers that are an arbitrary integer. Physically, this means that by traversing the boundary of the plane the spinor can wind around the order parameter an arbitrary number of times.

Similarly we can also discuss singular pointlike defects. Since the boundary of a three-dimensional gas is equivalent to the surface of a three-dimensional sphere, singular pointlike defects are determined by the second homotopy group $\pi_{2}(G)$ [13]. Because also $\pi_{2}\left(S^{1} \times S^{2}\right)=Z$, such topological excitations thus indeed exist in the case of a spin-1 Bose gas with antiferromagnetic interactions. In view of the work of 't Hooft and Polyakov we refer to these excitations as monopoles [14], although it would also be justifiable to call them singular Skyrmions. In contrast to the nonsingular Skyrmions in the Bose-Einstein ferromagnets, which inherently are nonequilibrium objects, the monopoles turn out to be thermodynamically stable excitations as we show next.

Monopole texture.- The grand-canonical energy of the spinor condensate can be obtained from the usual GrossPitaevskii theory, which, for the restricted parameter space given in Eq. (1), leads to the expression

$$
\begin{aligned}
E[n, \zeta]=\int d \mathbf{r} \psi^{*}(\mathbf{r})( & -\frac{\hbar^{2} \nabla^{2}}{2 m}+V_{\text {trap }}(\mathbf{r})-\mu \\
& +\frac{g_{n}}{2}|\psi(\mathbf{r})|^{2} \\
& \left.+\frac{\hbar^{2}}{2 m}[\nabla \mathbf{m}(\mathbf{r})]^{2}\right) \psi(\mathbf{r}),
\end{aligned}
$$

where $\psi(\mathbf{r})=\sqrt{n(\mathbf{r})} e^{i \vartheta(\mathbf{r})}$ is the superfluid order parameter, $V_{\text {trap }}(\mathbf{r})=m \omega^{2} \mathbf{r}^{2} / 2$ is an isotropic harmonic trapping potential, $\mu$ is the chemical potential, and $g_{n} \equiv$ $4 \pi a_{n} \hbar^{2} / m$ is the appropriate coupling constant for density fluctuations. Minimization of this energy determines both the spin texture $\mathbf{m}(\mathbf{r})$ and the density profile $n(\mathbf{r})$ of the monopole [15]. Since gradients in the spin texture do not couple to the superfluid phase $\vartheta(\mathbf{r})$, we can at this point already conclude that the presence of a monopole will not induce any superfluid flow in the atomic cloud. From now on we therefore no longer consider this degree of freedom.

Interestingly, the spin texture is uniquely determined by the fact that it should have a topological winding number [16]

$$
W=\frac{1}{8 \pi} \int d \mathbf{r} \epsilon_{i j k} \epsilon_{\alpha \beta \gamma} \partial_{i} m_{\alpha} \partial_{j} m_{\beta} \partial_{k} m_{\gamma}
$$

equal to 1 and that it should also minimize the gradient energy

$$
E_{\text {grad }}[n, \zeta]=\int d \mathbf{r} \frac{n(\mathbf{r}) \hbar^{2}}{2 m}[\nabla \mathbf{m}(\mathbf{r})]^{2} .
$$

As we discuss in more detail in a moment, the latter requires the spin texture to be as symmetric as possible. In combination with the first requirement, we thus conclude that $\mathbf{m}_{\mathrm{HP}}(\mathbf{r})=\mathbf{r} / r$ for a monopole in the center of the trap. Indeed, a spin texture with the same winding number can be obtained by rotating $\mathbf{m}_{\mathrm{HP}}(\mathbf{r})$ by an arbitrary rotation matrix $\mathbf{R}(r)$ that depends only on the radial distance $r$. As a result the gradient energy is turned into the suggestive form

$E_{\text {grad }}[n, \zeta]=\int d \mathbf{r} \frac{n(\mathbf{r}) \hbar^{2}}{2 m}\left|[\boldsymbol{\nabla}-i \mathbf{A}(\mathbf{r})] \mathbf{m}_{\mathrm{HP}}(\mathbf{r})\right|^{2}$,

which brings out even more clearly the analogy with the $O$ (3) gauge theory studied by 't Hooft and Polyakov. In our case the vector potential is, however, defined by $\mathbf{A}(\mathbf{r})=i\left(\mathbf{R}^{-1}(r) \nabla \mathbf{R}(r)\right)$ and is always orthogonal to the gradient of $\mathbf{m}_{\mathrm{HP}}(\mathbf{r})$. The gradient energy is therefore minimized for $\mathbf{R}(r)=\mathbf{1}$, as anticipated previously.

Substituting our hedgehog solution $\mathbf{m}_{\mathrm{HP}}(\mathbf{r})$ for the spin texture into Eq. (2), we see that the density profile of the spinor condensate is found from a Gross-Pitaevskii equation with a centrifugal barrier equal to $\hbar^{2} / m r^{2}$. Considering first the homogeneous case and writing the superfluid order parameter as $\psi(\mathbf{r})=\left(\mu / g_{n}\right)^{1 / 2} f(r / \xi)$, with $\xi=\left(\hbar^{2} / 2 m \mu\right)^{1 / 2}$ the correlation length, we obtain explicitly that

$$
\left(-\frac{1}{\rho^{2}} \frac{d}{d \rho}\left(\rho^{2} \frac{d}{d \rho}\right)+\frac{2}{\rho^{2}}+f^{2}(\rho)-1\right) f(\rho)=0 .
$$

We have not been able to solve this equation analytically, but its numerical solution is shown in Fig. 1. What is most important for our purposes, however, is the large distance behavior of the density profile. Neglecting the gradient terms in the left-hand side, we easily find that $f(\rho) \simeq 1-$ $1 / \rho^{2}$ for $\rho \gg 1$. Furthermore, the monopole is clearly seen to possess a core with a typical size of the order of the correlation length. Inside this core the density is strongly reduced, which offers the opportunity to detect monopoles by the same expansion experiments that have recently also been used to observe vortices [17].

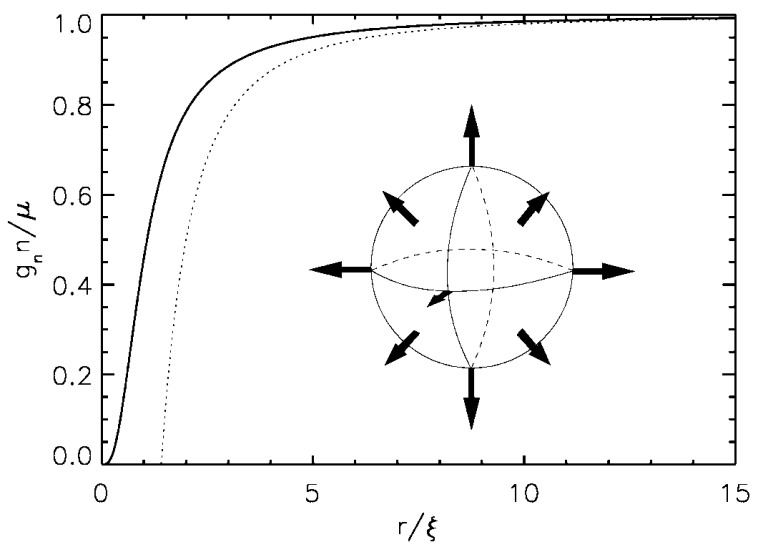

FIG. 1. Spin texture and density profile of the 't Hooft-Polyakov monopole. The solid line shows the exact numerical result, whereas the dashed line shows the analytic long distance behavior $n(r) \simeq\left(\mu / g_{n}\right)\left[1-2(\xi / r)^{2}\right]$. 
Having obtained the spin texture and the density profile of the monopole, we are now in a position to determine also its energy. Placing the monopole in the center of a spherical volume with a large radius $R \gg \xi$, we obtain

$$
E_{\mathrm{HP}}=\frac{\mu}{a_{n}}\left(R-R_{\text {core }}\right),
$$

where $R_{\text {core }}$ is the effective core size of the monopole. Its calculation requires knowledge of the complete density profile and we find numerically that $R_{\text {core }} \simeq 1.4 \xi$. The monopole energy thus diverges linearly with the system size, which implies that in the thermodynamic limit only pairs of monopoles with opposite winding numbers require a finite energy for their creation.

The physical interpretation of Eq. (7) is that to calculate the monopole energy we need to evaluate only the gradient energy for a fixed density equal to $\mu / g_{n}$, but restrict the integration to the volume outside a spherical core region with radius $R_{\text {core }}$. This interpretation is particularly useful for a trapped spinor condensate in the Thomas-Fermi limit, when the size $R_{\mathrm{TF}}=\left(2 \mu / m \omega^{2}\right)^{1 / 2}$ of the condensate is much larger than the correlation length $\xi$. For a monopole at position $\mathbf{u}$ near the center of the trap, we find in this manner that

$$
E_{\mathrm{HP}}[\mathbf{u}] \simeq \frac{2 \mu}{3 a_{n}} R_{\mathrm{TF}}\left(1-\frac{\mathbf{u}^{2}}{R_{\mathrm{TF}}^{2}}+\frac{\mathbf{u}^{4}}{5 R_{\mathrm{TF}}^{4}}+\cdots\right),
$$

if we neglect the core contributions that are smaller by a factor of $\xi / R_{\mathrm{TF}}$. The significance of this result will become clear once we understand the dynamical properties of the monopole.

Monopole dynamics. - In the first instance we expect the dynamics of the monopole to be determined by the action $S[n, \zeta]=\int d t(T[n, \zeta]-E[n, \zeta])$ with a time-derivative term that is equal to

$$
T[n, \zeta]=\int d \mathbf{r} n(\mathbf{r}, t) \zeta^{\dagger}(\mathbf{r}, t) i \hbar \frac{\partial}{\partial t} \zeta(\mathbf{r}, t)
$$

in the Gross-Pitaevskii theory. However, when we restrict ourselves to the antiferromagnetic spinor $\zeta_{\mathrm{AF}}(\mathbf{r}, t)$, the time-derivative term in the action exactly vanishes due to the normalization condition $\mathbf{m}^{2}(\mathbf{r}, t)=1$. To find any dynamics for the monopole we thus need to consider also fluctuations that bring the spinor condensate out of the antiferromagnetic order parameter space. In the Thomas-Fermi limit, the relevant dynamical part of the action thus becomes

$$
\begin{aligned}
S_{\mathrm{dyn}}[n, \zeta]=\int d t( & T[n, \zeta] \\
& \left.-\int d \mathbf{r} \frac{g_{s}}{2}[n(\mathbf{r}, t)\langle\mathbf{F}\rangle(\mathbf{r}, t)]^{2}\right),
\end{aligned}
$$

with $g_{s} \equiv 4 \pi a_{s} \hbar^{2} / m$ the appropriate coupling constant for spin-density fluctuations. Using this action we can now investigate the effect of the above-mentioned fluctuations by substituting $\zeta(\mathbf{r}, t)=\zeta_{\mathrm{AF}}(\mathbf{r}, t)+\delta \zeta(\mathbf{r}, t)$ and expanding the action up to quadratic order in $\delta \zeta(\mathbf{r}, t)$. Solving then the Euler-Lagrange equation for $\delta \zeta(\mathbf{r}, t)$ and substituting the solution back into the action, we ultimately find the desired low-frequency result [18]

$$
S_{\mathrm{dyn}}[n, \zeta]=\int d t \int d \mathbf{r} \frac{\hbar^{2}}{2 g_{s}}\left(\frac{\partial \mathbf{m}(\mathbf{r}, t)}{\partial t}\right)^{2} .
$$

While performing the calculation, we must make sure that we are not considering fluctuations of the spinor within the antiferromagnetic order parameter space. This requires the matrix elements $\zeta_{\mathrm{AF}}^{\dagger}(\mathbf{r}, t) \mathbf{F} \delta \zeta(\mathbf{r}, t)$ to be real, because for fluctuations within the antiferromagnetic parameter space we have in lowest order that $\mathbf{0}=\langle\mathbf{F}\rangle(\mathbf{r}, t)=$ $\zeta_{\mathrm{AF}}^{\dagger}(\mathbf{r}, t) \mathbf{F} \delta \zeta(\mathbf{r}, t)+\delta \zeta^{\dagger}(\mathbf{r}, t) \mathbf{F} \zeta_{\mathrm{AF}}(\mathbf{r}, t)$. Moreover, the normalization of the spinor requires also that $\zeta_{\mathrm{AF}}^{\dagger}(\mathbf{r}, t) \delta \zeta(\mathbf{r}, t)=0$.

The importance of this result is twofold. First, from Eqs. (4) and (10) we see that at the quantum level the dynamics of the space-independent part of the vector field $\mathbf{m}(\mathbf{r})$ is governed by the following time-dependent Schrödinger equation:

$$
i \hbar \frac{\partial}{\partial t} \Psi(\mathbf{m}, t)=-\frac{\hbar^{2}}{2 I} \nabla_{\mathbf{m}}^{2} \Psi(\mathbf{m}, t)
$$

for the wave function $\Psi(\mathbf{m}, t)$. It thus corresponds exactly to a quantum rotor with a moment of inertia equal to $I=\hbar^{2} V_{0}(\mu) / g_{s}$, where $V_{0}(\mu)$ is the total volume of the spinor condensate in the Thomas-Fermi limit. In an harmonic trap the moment of inertia is thus proportional to the $3 / 5$ power of the total number of atoms. The eigenstates of this Schrödinger equation are the spherical harmonics $Y_{S, M_{S}}(\mathbf{m})$. In this way we thus recover the fact that according to quantum mechanics both the total spin of the Bose-Einstein antiferromagnet as well as its projection on the quantization axis must always be an integer. More precisely, since the ground state wave function is given by $Y_{0,0}(\mathbf{m})=1 / \sqrt{4 \pi}$, we have actually shown that the many-body wave function of the antiferromagnetic spinor condensate is a singlet state exactly $[7,8]$. Note that physically this phenomenon is equivalent to the way in which "diffusion" of the overall phase of a Bose-Einstein condensate leads to the conservation of particle number $[19,20]$. The main difference is that here the diffusion takes place on the surface of a unit sphere instead of on a unit circle.

Second, and most important for our purposes, we can now determine the single monopole dynamics, by using the ansatz $\mathbf{m}(\mathbf{r}, t)=\mathbf{m}_{\mathrm{HP}}[\mathbf{r}-\mathbf{u}(t)]$ for the texture of a moving monopole, which is expected to be accurate for small velocities $d \mathbf{u}(t) / d t$ and, in the inhomogeneous case, near the center of the trap where $u / R_{\mathrm{TF}} \ll 1$. Substituting this ansatz into Eq. (11) and remembering also Eq. (8), we find that the action for the center-of-mass motion of the monopole becomes precisely that of a massive particle 


$$
S_{\mathrm{HP}}[\mathbf{u}]=\int d t\left(\frac{m_{\mathrm{HP}}}{2}\left(\frac{d \mathbf{u}(t)}{d t}\right)^{2}-E_{\mathrm{HP}}[\mathbf{u}]\right)
$$

with a mass given by $m_{\mathrm{HP}}=2 m R_{\mathrm{TF}} / a_{s}$. The semiclassical equation of motion for the monopole position is therefore simply Newton's equation

$$
m_{\mathrm{HP}} \frac{d^{2} \mathbf{u}(t)}{d t^{2}}=-\nabla_{\mathbf{u}} E_{\mathrm{HP}}[\mathbf{u}] .
$$

In view of the fact that the energy of the monopole decreases as the distance to the center of the trap increases, we conclude that in general the monopole is always accelerated to the boundary of the condensate. Typically it will reach that boundary in a time interval of order

$$
\Delta t_{\mathrm{HP}} \simeq \frac{\pi}{2} \sqrt{\frac{3 m R_{\mathrm{TF}}^{2}}{2 \mu} \frac{a_{n}}{a_{s}}}=\frac{\pi}{2 \omega} \sqrt{\frac{3 a_{n}}{a_{s}}},
$$

which is typically about $1 / \omega$ and therefore sufficiently long for present-day experiments to be able to observe the monopole once it is created [21]. In this context it should be noted that all our calculations are performed at zero temperature. In the presence of a normal component, the monopole experiences damping, which increases the time needed to reach the edge of the spinor condensate.

To summarize, we have investigated the most important equilibrium and nonequilibrium properties of a monopole in a trapped, antiferromagnetic Bose-Einstein condensate. A further direction of research is an ab initio calculation of the above-mentioned friction force on the monopole. In fact, we expect the thermal cloud to lead not only to dissipation but also to noise. Both effects can be conveniently treated within the general framework of the stochastic field theory that was developed previously for nonequilibrium phenomena in partially Bose-Einstein condensed gases [20]. Another interesting topic is the interaction between two monopoles and the many-body properties of a gas of these topological objects. Finally, a very important experimental problem is the creation of a monopole. Of course, pairs of monopoles with opposite winding numbers can in principle be created in a thermal quench or by sufficiently shaking up the spinor condensate. However, a more controlled creation mechanism is desirable. After submission of this Letter, such a creation mechanism was indeed proposed by Martikainen and Suominen [22].
These authors find that a combination of well established phase-imprinting methods $[4,21]$ is sufficient to create an excited state of the spinor Bose-Einstein condensate that ultimately relaxes to a state containing a single monopole.

This work is supported by the Stichting voor Fundamenteel Onderzoek der Materie (FOM), which is financially supported by the Nederlandse Organisatie voor Wetenschappelijk Onderzoek (NWO).

[1] D. M. Stamper-Kurn et al., Phys. Rev. Lett. 80, 2027 (1998).

[2] H.-J. Miesner et al., Phys. Rev. Lett. 82, 2228 (1999); D. M. Stamper-Kurn et al., Phys. Rev. Lett. 83, 661 (1999).

[3] C. J. Myatt et al., Phys. Rev. Lett. 78, 586 (1997).

[4] M. R. Matthews et al., Phys. Rev. Lett. 83, 2498 (1999); M. R. Matthews et al., Phys. Rev. Lett. 83, 3358 (1999).

[5] T.-L. Ho, Phys. Rev. Lett. 81, 742 (1998).

[6] T. Ohmi and K. Machida, J. Phys. Soc. Jpn. 67, 1822 (1998).

[7] C. K. Law, H. Pu, and N. P. Bigelow, Phys. Rev. Lett. 81, 5257 (1998).

[8] T.-L. Ho and S.-K. Yip, Phys. Rev. Lett. 84, 4031 (2000).

[9] S.-K. Yip, Phys. Rev. Lett. 83, 4677 (1999).

[10] S. Tuchiya and S. Kurihara, cond-mat/0101109.

[11] U. Al Khawaja and H. T.C. Stoof, Nature (London) 411, 918 (2001).

[12] G. 't Hooft, Nucl. Phys. B79, 276 (1974); A. M. Polyakov, JETP Lett. 20, 194 (1974).

[13] N. D. Mermin, Rev. Mod. Phys. 51, 591 (1979).

[14] Note that the monopoles discussed by T. Busch and J. R. Anglin, Phys. Rev. A 60, R2669 (1999), and J. J. GarcíaRipoll et al., Phys. Rev. A 61, 053609 (2000), exist only in two dimensions since $\pi_{2}(\mathrm{SO}(3))=0$.

[15] We call the vector field $\mathbf{m}(\mathbf{r})$ a spin texture even though its effect is visible only in the spin fluctuations, i.e., for example, in $\left\langle F_{\alpha} F_{\beta}\right\rangle(\mathbf{r})=\delta_{\alpha \beta}-m_{\alpha}(\mathbf{r}) m_{\beta}(\mathbf{r})$.

[16] R. Rajaraman, Solitons and Instantons (North-Holland, Amsterdam, 1982).

[17] K. W. Madison et al., Phys. Rev. Lett. 84, 806 (2000).

[18] This result was obtained previously using different methods by F. Zhou, cond-mat/0102372.

[19] M. Lewenstein and L. You, Phys. Rev. Lett. 77, 3489 (1996).

[20] H. T. C. Stoof, J. Low Temp. Phys. 114, 11 (1999).

[21] See, for example, S. Burger et al., Phys. Rev. Lett. 83, 5198 (1999), and J. Denschlag et al., Science 287, 97 (2000), for a similar situation.

[22] J.-P. Martikainen and K.-A. Suominen, cond-mat/0106301. 